\title{
Assessment of testes with two-dimensional Shear Wave Elastography in patients with operated inguinal hernia
}

\author{
Mehmet Sedat Durmaz ${ }^{1}$, Fatih Ateş ${ }^{1}$, Serdar Arslan², Turgay Kara ${ }^{3}$, Funda Gökgöz Durmaz ${ }^{4}$, \\ Mehmet Ali Eryılmaz ${ }^{5}$ Kemal Arslan 5
}

${ }^{1}$ Selçuk University, Medicine Faculty, Department of Radiology, Konya, ${ }^{2}$ Istanbul University, Cerrahpasa Medicine Faculty, department of Radiology, Istanbul, ${ }^{3} \mathrm{Health}$ Sciences University, Training and Research Hospital, Department of Radiology, Konya, ${ }^{4}$ Karatay Community Health Center Family Medicine Konya, ${ }^{5}$ Health Sciences University, Training and Research Hospital, Department of General Surgery, Konya, Turkey

\begin{abstract}
Aim: We compared the two-dimensional shear-wave elastography (2D-SWE) values between the testes with same side operated inguinal hernia (IH) and the contralateral testes, as well as the testes of healthy volunteers without IH. Material and methods: A total of 189 participants (117 unilateral [117 testes] and 8 bilateral operated IH patients [16 testes] and 64 healthy volunteers [128 testes]), providing a total of 378 testicles, were investigated prospectively. All patients underwent B-mode ultrasonography (US) and 2D-SWE examinations. Operation type, the period between diagnosis and operation, the period since the operation, testes volumes, and 2D-SWE values were compared. Results: The B-mode US finding of the testes were normal in all participants. The mean testes' volume of same side operated IH was significantly lower comparing to contralateral testes and the healthy group $(\mathrm{p}<0.001)$. The $2 \mathrm{D}-\mathrm{SWE}$ values of the testes with same side operated IH were significantly higher comparing to the contralateral testes and the healthy group $(\mathrm{p}<0.001)$. There was a statistically significant correlation between 2D-SWE values and IH severity, as well as the duration of the hernia $(\mathrm{p}=0.001)$. There was no significant correlation between the IH severity and testes volume $(\mathrm{p}=0.285)$. No significant difference was found between the direct and indirect IH in terms of testicular volume and SWE values and between the duration of the hernia, the time after sugery, testicular volume and SWE values according to operation techniques ( $p>0.005)$. Conclusions: The 2D-SWE can be used as an effective imaging method to evaluate testicular stiffness with objective numerical values, to estimate the severity of histologic damage in patients with operated IH.
\end{abstract}

Keywords: inguinal hernia; Shear Wave Elastography; testes; ultrasonography

\section{Introduction}

Inguinal hernia $(\mathrm{IH})$ is an important public health problem that requires surgical treatment and has an incidence rate of approximately 5 to $10 \%[1,2]$. There are

Received 12.09.2020 Accepted 15.12.2020

Med Ultrason

2021, Vol. 23, No 2, 181-187

Corresponding author: Fatih Ateş MD

Selçuk University, Medicine Faculty, Department of Radiology, Konya, Turkey

313 Ardıclı Mahallesi, Celal Bayar Cad.,

Selçuklu, 42250 Konya, Turkey

Phone: +90 5468375296

Fax: +903325121653

E-mail: fatih_ates81@hotmail.com two main subtypes of $\mathrm{IH}$, direct and indirect, the last being more common [3]. IH can lead to serious complications, including testicular damage and atrophy resulting from the narrowing of the blood vessels in the spermatic cord [4]. Testicular damage due to IH surgery has been well documented being reported to range from 1 to $14 \%$ [3]. The testicular blood supply may return to normal as the pressure effect of the hernia-induced spermatic cord is removed after the operation $[5,6]$. However, testicular damage can be caused by injury to the spermatic cord and vas deferens during surgery, postoperative infection, and foreign body inflammatory responses to the mesh, scar, and granulation tissue [7-9].

Progressive histopathological changes, such as the reduction of the germ cell count, delay germ cell matura- 
tion and atrophy can occur due to the effects of the $\mathrm{IH}$ and possible postoperative changes in the inguinal area [10]. As a consequence, the testis becomes stiffer due to parenchymal fibrosis [11]. Ultrasonography (US) is a commonly used imaging technique to assess testicular pathologies. Testicular morphology, volume, and vascularity can be evaluated with US. However, US results may indicate no testicular damage even though histological testicular damage is present $[11,12]$. Testicular biopsy is the gold standard technique for evaluating histological damage. However, this is no longer recommended because of the high likelihood that the procedure will lead to complications [11].

Two-dimensional shear wave elastography (2DSWE), a US imaging technique that provides quantitative measurement of tissue stiffness, provides an alternative technique for estimating histopathological abnormalities that cannot be detected by conventional US [11]. The 2D-SWE can evaluate the testis stiffness to estimate the level of fibrosis resulting from damage has been reported $[11,12]$. Parenchymal damage in the testis could be assessed using a quantitative evaluation of the degree of parenchymal fibrosis caused by IH and possible postoperative changes in the inguinal area.

In this study, we quantitatively evaluated the stiffness of testes parenchyma using 2D-SWE on the same side as the IH operation and we compared the results with normal testes. To the best of our knowledge, no previous studies have focused on the evaluation of posibile testicular damage in patients with operated IH using the 2D-SWE technique.

\section{Materials and methods}

This study was conducted between December 2017 and January 2020, after the approval of the local research Ethics Committee. All subjects were informed about the study protocol and written informed consent was obtained before the procedure.
A total of 189 participants and 378 testes (1:1 ratio of left to right) were included in the study. Group A, totalling 133 testes, was composed of 117 patients who had undergone unilateral IH surgery (117 testes) and 8 patients who had undergone bilaterally IH surgery (16 testes). Group A was divided into three subgroups according to the hernia repair operation technique applied: preperitoneal alloplasty with a posterior open inguinal approach (Kugel); open mesh hernia repair (Lichtenstein); and endoscopic totally extraperitoneal (TEP) mesh hernia repair. There were no reported intraoperative complications in this group of patients. Group A was further subdivided into IH types, either direct or indirect, according to the surgical findings, and the level of IH location (proximal [grade I], middle [grade II] and distal inguinal canal, almost in scrotum [grade III]). The duration of the hernia in these patients and the period between the IH operation and the US examination were recorded. Group B was composed of 117 testes located on the opposite side of the site of IH surgery. Group C was composed of 128 testes from 64 healthy volunteers. The groups characteristics are summarized in Table I.

Patients with any of the following traits or conditions were excluded from the study: less than 18 years of age; a history of any lower abdominal-pelvic surgery or orchiectomy; newly diagnosed IH; recurrent or failed $\mathrm{IH}$ repair; previously emergency exploration for complications of IH (e.g., irreducible hernia, bowel obstruction, and strangulation); previous chemotherapy/radiotherapy treatment; previous abnormal US findings, such as focal lesion in the testicle or epididymis, varicocele, hydrocele, epididymitis, and orchitis.

\section{US and 2D-SWE}

All participants underwent B-mode US and 2D-SWE examinations of the testes in the supine position. The US and 2D-SWE examinations were performed with a highfrequency (4-14 MHz) linear array transducer (Aplio 500, Canon Medical System Corporation, Tustin, CA). Each patient was examined under the same standard cir-

Table I. Number of testes included into the study, according to grouping of testes.

\begin{tabular}{|c|c|c|c|c|c|}
\hline \multicolumn{5}{|l|}{ Classification } & \multirow{2}{*}{$\frac{\text { Nr. of testes }}{133(35.19 \%)}$} \\
\hline $\begin{array}{l}\text { Symptomatic } \\
\text { group }\end{array}$ & $\begin{array}{l}\text { Inguinal canal proximal } \\
\text { (grade 1) (14) }\end{array}$ & Direct IH (33) & Lichtenstein operation history (80) & Group A & \\
\hline \multirow[t]{2}{*}{$\begin{array}{l}\text { History of } \\
\text { operation } \\
\text { for IH }\end{array}$} & $\begin{array}{l}\text { Inguinal canal middle } \\
\text { (grade 2) }(60)\end{array}$ & Indirect IH (100) & TEP operation history (35) & & \\
\hline & $\begin{array}{l}\text { Inguinal canal distal } \\
\text { or scrotal (grade 3) (59) }\end{array}$ & & Kugel operation history (18) & & \\
\hline \multirow{2}{*}{$\begin{array}{l}\text { Asymptomatic } \\
\text { group }\end{array}$} & \multicolumn{2}{|c|}{ Normal opponent of IH operation testicle } & & Group B & $117(30.95 \%)$ \\
\hline & \multicolumn{2}{|l|}{ Bilateraly normal testes } & & Group C & $128(33.86 \%)$ \\
\hline
\end{tabular}

Inguinal hernia (IH), endoscopic totally extraperitoneal (TEP). 


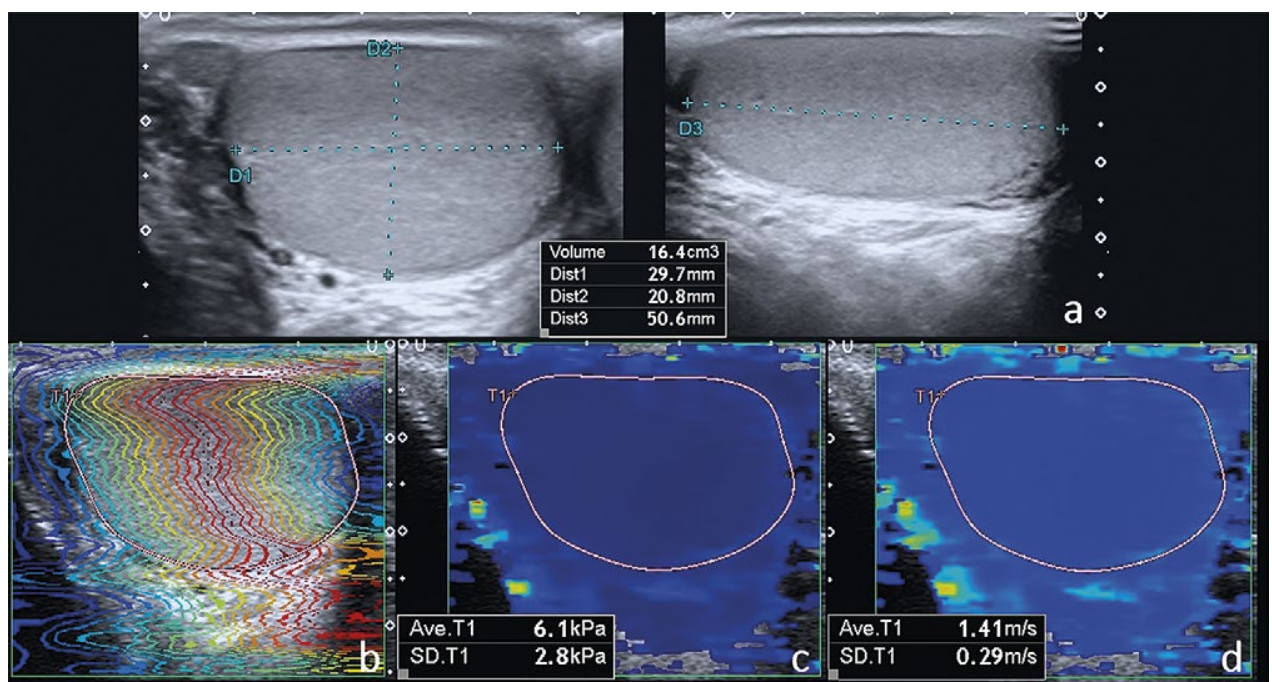

Fig 1. A 52-year-old male healthy volunteer. The right testis was measured in three dimensions and testes volume was calculated as $16.4 \mathrm{~cm}^{3}$. The B-mode US findings of the testis were in normal parenchymal structure (a). Quantitative 2D-SWE values were measured in the transverse plane at the level of the testicular hilus by drawing manually contours of the entire testis structure on propagation mode with a free ROI (b). The quantitative elasticity values were measured as $6.1 \mathrm{kPa}(\mathrm{c})$ and $1.41 \mathrm{~m} / \mathrm{s}(\mathrm{d})$.

cumstances. US and 2D-SWE were carried out by a radiologist with 15 years' experience using US and six years of experience using 2D-SWE. The pulse repetition frequency, focal zone, gain and wall filter were adjusted to obtain the optimal image for each participant. The inguinal region was evaluated for the presence or recurrence of hernia in all participants before evaluating the testes. The examination of the testes was started with a B-mode US. Each testicle was measured in three dimensions. Testes volume was calculated by US device software (testicular volume $=$ width $\times$ height $\times$ depth $\times 10^{3} \times$ $0.523)$.

Quantitative 2D-SWE measurements were taken for each participant in the transverse plane at the level of the testicular hilus, using a magnified view as much as possible, by manually drawing the contours of the entire testis structure using a free region of interest (ROI) in all testes (fig 1). Three images of the testes were obtained by placing the US probe very lightly on the scrotum and then the average of these three measurements was calculated as the SWE value of the testis. The quantitative elasticity values were measured in both meters/second $(\mathrm{m} / \mathrm{s})$ and kilopascal (kPa).

The A, B, and C groups were compared in terms of the 2D-SWE values and testes volume. Among the testes in Group A, we assessed the correlation between 2D-SWE values and the duration of hernia and the period since the inguinal hernia operation. Furthermore, 2D-SWE values were compared among Group A patients according to the type of hernia and surgical procedure, and also the grade of hernia.

\section{Statistical analysis}

The Statistical Package for Social Sciences (SPSS) version 22.0 (Inc., Chicago, Illinois, USA) software was used to evaluate the data. The descriptive statistics were expressed as mean, SD, minimum-maximum values, frequency, and percentile. One-way analysis of variance (ANOVA) and Student's t-test were used to evaluate the differences between the groups. The normality assumption for the distribution of continuous variables was tested using the Kolmogorov-Smirnov test. Pearson's correlation analysis was used to evaluate the relationship between 2D-SWE values and the degree of hernia, duration of hernia, and the period since the operation. A p-value of less than 0.05 was accepted as statistically significant.

\section{Results}

The mean age, testis volume and 2D-SWE values for each of the three groups (A, B, and C) are summarized in Table II. There was no statistically significant difference in the mean age among the three groups $(p=0.406)$. The B-mode US of the testes were normal in all participants and no recurrent-failed repair of IH in those who underwent IH surgery was observed. There was a statistically significant difference between both testes' volume and 2D-SWE values $(\mathrm{kPa}$ and $\mathrm{m} / \mathrm{s})$ across the three groups. A post hoc Tukey test indicated that the mean testes' volume was significantly lower and 2D-SWE values were significantly higher in group A comparing with Groups $\mathrm{B}$ and $\mathrm{C}$. 
There were no statistically significant differences between groups $\mathrm{B}$ and $\mathrm{C}$ in terms of testes volume and 2DSWE values (Table III).

There was no significant difference between the duration of the hernia, the period passed after the IH operation, testicular volume and SWE values according to operation techniques ( $p>0.005)$. In addition, there was no significant difference between the direct and indirect $\mathrm{IH}$ with respect to age, duration of hernia, the period since the IH operation, testicular volume and SWE values ( $\mathrm{p}>0.005$, Table IV).

IH was located in proximal (grade I), middle (grade II) and distal, almost in scrotum (grade III) in $14(10.5 \%)$, $60(54.5 \%)$ and $59(44.4 \%)$ patients, respectively. There was no statistically significant difference among these three groups in terms of age, duration of hernia and the period since the IH operation ( $p>0.005)$. There was a significant positive correlation between the degree of hernia and 2D-SWE values of testes for both $\mathrm{kPa}$ and $\mathrm{m} / \mathrm{s}$ $(\mathrm{r}=0.643, \mathrm{p}=0.001$ and $\mathrm{r}=0.614, \mathrm{p}=0.001$, respectively). There was no correlation between the grade of hernia and testes volume $(\mathrm{r}=-0.093, \mathrm{p}=0.285)$.

There was a positive correlation between the duration of the hernia and 2D-SWE values for both $\mathrm{kPa}$ and $\mathrm{m} / \mathrm{s}$ $(\mathrm{r}=0.806 \mathrm{p}=0.001$ and $\mathrm{r}=0.736, \mathrm{p}=0.001$, respectively $)$. There was no significant correlation between the duration of hernia and testis volume $(r=-0.004, p=0.965)$ and between the time after the IH operation, 2D-SWE values for both $\mathrm{kPa}$ and $\mathrm{m} / \mathrm{s}(\mathrm{r}=0.113, \mathrm{p}=0.197$ and $\mathrm{r}=0.081, \mathrm{p}=0.351$, respectively) and testes volume $(\mathrm{r}=0.014, \mathrm{p}=0.869)$.

\section{Discussion}

This study focuses on whether IH or surgery to repair IH has any effect on testicular stiffness and testicular volume. We found that on the side of IH surgery the testes were smaller and stiffer. The stiffness of testes was correlated with the grade of $\mathrm{IH}$ and duration of hernia but no significant correlation was found between testicular volume and grade of IH or the duration of the hernia. Our results suggest that SWE can be used as an effective imaging technique for assessing testicular damage in these patients. The significant correlation between 2DSWE values and the degree and the duration of the hernia supports the hypothesis that the degree of testicular damage changes according to the effect of pressure and the duration of the pressure on the spermatic cord and vas deferens.

Table II. The mean age, testes volume and 2D-SWE values of testes according to the group which we used.

\begin{tabular}{|c|c|c|c|c|c|c|}
\hline & & Totaly & Group A & Group B & Group C & $\mathbf{p}$ \\
\hline Age (year) & & $45.50 \pm 11.20$ & $48.11 \pm 11.55$ & $47.72 \pm 11.62$ & $40.64 \pm 8.60$ & 0.406 \\
\hline Volume $\left(\mathrm{cm}^{3}\right)$ & & $15.07 \pm 4.21$ & $13.60 \pm 3.83$ & $15.71 \pm 4.58$ & $15.97 \pm 3.80$ & 0.001 \\
\hline \multirow{2}{*}{$\begin{array}{l}\text { Mean 2D-SWE } \\
\text { value }\end{array}$} & $\mathrm{kPa}$ & $7.04 \pm 1.81$ & $8.54 \pm 2.08$ & $6.22 \pm 1.08$ & $6.30 \pm 0.90$ & 0.001 \\
\hline & $\mathrm{m} / \mathrm{s}$ & $1.44 \pm 0.16$ & $1.56 \pm 0.16$ & $1.37 \pm 0.10$ & $1.38 \pm 0.13$ & 0.001 \\
\hline
\end{tabular}

Two-dimensional shear-wave elastography (2D-SWE), inguinal hernia $(\mathrm{IH})$, kilopascal $(\mathrm{kPa})$ and meters/second $(\mathrm{m} / \mathrm{s})$.

Table III. The p value when the Group A, B and C were compared between each other acording to testis volume and 2D-SWE values.

\begin{tabular}{llllll}
\hline & & A versus B & A versus C & A versus (B+C) & B versus C \\
\hline Testes volume & & 0.001 & 0.001 & 0.001 & 0.622 \\
Mean 2D-SWE & $\mathrm{kPa}$ & 0.001 & 0.001 & 0.001 & 0.520 \\
value & $\mathrm{m} / \mathrm{s}$ & 0.001 & 0.001 & 0.001 & 0.607 \\
\hline
\end{tabular}

Two-dimensional shear-wave elastography (2D-SWE), inguinal hernia (IH), kilopascal (kPa) and meters/second (m/s).

Table IV. The mean age, volume and 2D-SWE values of testes in Group A according to type of inguinal hernia and operation type

\begin{tabular}{|c|c|c|c|c|c|c|c|c|}
\hline \multirow{2}{*}{\multicolumn{2}{|c|}{ Age (year) }} & Direct IH & Indirect IH & $\mathbf{p}$ & Lichtenstein & TEP & Kugel & $\mathbf{p}$ \\
\hline & & $49.39 \pm 9.13$ & $47.69 \pm 12.25$ & 0.465 & $47.27 \pm 13.14$ & $50.37 \pm 9.73$ & $47.44 \pm 5.43$ & 0.543 \\
\hline \multicolumn{2}{|c|}{ Duration of IH (month) } & $4.90 \pm 2.33$ & $4.40 \pm 2.33$ & 0.280 & $4.71 \pm 2.23$ & $4.14 \pm 2.14$ & $4.44 \pm 3.09$ & 0.297 \\
\hline \multicolumn{2}{|c|}{ Time from surgery (month) } & $4.27 \pm 2.21$ & $4.23 \pm 2.44$ & 0.929 & $3.78 \pm 2.28$ & $5.37 \pm 2.17$ & $4.05 \pm 2.55$ & 0.828 \\
\hline \multicolumn{2}{|c|}{ Testes volume $\left(\mathrm{cm}^{3}\right)$} & $14.24 \pm 3.51$ & $13.39 \pm 3.92$ & 0.268 & $13.61 \pm 4.01$ & $13.41 \pm 3.86$ & $13.95 \pm 3.06$ & 0.893 \\
\hline \multirow{2}{*}{$\begin{array}{l}\text { Mean 2D-SWE } \\
\text { value }\end{array}$} & $\mathrm{kPa}$ & $9.02 \pm 2.51$ & $8.38 \pm 11.90$ & 0.122 & $8.67 \pm 2.03$ & $8.26 \pm 1.29$ & $8.46 \pm 3.28$ & 0.620 \\
\hline & $\mathrm{m} / \mathrm{s}$ & $1.61 \pm 0.20$ & $1.54 \pm 0.14$ & 0.080 & $1.58 \pm 0.16$ & $1.54 \pm 0.11$ & $1.54 \pm 0.21$ & 0.414 \\
\hline
\end{tabular}

Two-dimensional shear-wave elastography (2D-SWE), inguinal hernia (IH), endoscopic totally extraperitoneal (TEP), kilopascal (kPa) and meters $/$ second $(\mathrm{m} / \mathrm{s})$. 
Since the effect of pressure is removed after the operation, the testicular damage can be prevented by returning the testicular blood supply to normal $[6,13]$. However, IH repair operations can lead to complications, which may arise during surgery or during the postoperative period that may cause damage to the spermatic cord and vas deferens [14]. Moreover, a foreign body inflammatory response to the mesh may result in testicular damage by causing fibrosis in the tissues surrounding the spermatic cord and vas deferens [7-9,14]. The incidence of testicular damage following IH surgery has been reported to range from 1 to 14\% [3]. Thus, both IH and IH repair surgery can cause testicular damage [14].

Assessment of damage to the testicle with serum hormone levels and sexual function is not reliable, especially in cases where unilateral IH is present [12]. B-mode US is the most commonly used initial imaging technique in the diagnosis of testicular damage, but B-mode US cannot provide sufficient information for a reliable diagnosis in every case and, in particular, has low sensitivity for evaluating testicular damage [14,15]. Testicular atrophy and a decrease in testicular volume is an important indicator of testicular damage in patients with $\mathrm{IH}$ and repaired IH, with a reported incidence of $0.5 \%$ [16]. Some studies have evaluated the effectiveness of IH on testicular perfusion with Doppler US using a resistive index (RI) $[15,17]$. These studies have shown the testis with $\mathrm{IH}$ has a significantly higher RI [18] or that there is no significant difference in the RI values [14,15,19-21]. Studies have also shown that there is no significant alteration of testicular perfusion after IH operation using Doppler US $[21,22]$. These results suggest that Doppler US and RI are not reliable to evaluate testicular damage. In our study, B-mode US aspect of the testicles was normal in all cases. However, the statistically significant higher testicular stiffness values obtained by 2D-SWE in those patients who had undergone IH surgery showed testicular damage. For these reasons, the efficacy of B-mode US in the assessment of testicular damage is limited. In our study, the testes volumes were significantly lower on the side of IH surgery. This finding supports that testicular volume may be used for the evaluation of testicular damage. However, there was a lack of a significant correlation between testicular volume and the grade of hernia and duration of hernia, suggesting that taken separately, the testicular volume cannot be used to assess testicular damage reliably.

The effectiveness of 2D-SWE to evaluate stiffness for estimating the degree of fibrosis as a result of testicular damage has been shown in many studies $[11,12,23]$. In these studies, SWE measurements were made in limited areas with ROI in constant small circle or square forms. This method of evaluation limits the repeatability and reduces the reliability of SWE values [11]. We used the average SWE values obtained by drawing the whole testis parenchyma with free ROI in the transverse plane. We consider this measurement method superior, more applicable, reliable and more repeatable than other methods used, in diagnosis and follow-up, under daily out-patient practice. A single study evaluated the testicles of patients with postoperative IH using compressive elastography and showed that testicle stiffness was higher on the operated side due to testicular damage [24]. The shortcomings of compressive elastography are its operator dependency and the semi-quantitative nature of the data, which may limit its reproducibility. However, 2DSWE is less operator-dependent, real-time, reproducible and allows the quantitative evaluation of the target tissue $[11,12,23]$. Elastic value $\mathrm{E}(\mathrm{kPa})$ is calculated using the equation $E=3 \rho(\mathrm{m} / \mathrm{s})^{2}$ ( $\rho$ refers to the tissue density, with approximated value in human body as $1 \mathrm{~g} / \mathrm{cm}^{3}$ and $\mathrm{m} / \mathrm{s}$ refers to the shear wave propagation velocity) [25]. The quantitative elasticity values both measured in $\mathrm{kPa}$ and $\mathrm{m} / \mathrm{s}$ revealed good diagnostic performance, but the specifity of the standart deviation and area under the ROC curve of the measurement in $\mathrm{kPa}$ were found significantly higher compared to the measurement in $\mathrm{m} / \mathrm{s}$ [26]. Since both SWE values $(\mathrm{m} / \mathrm{s}$ and $\mathrm{kPa})$ could be obtained with our US device, we measured both in our study. Thus, we think that our findings can be compared with future studies using $\mathrm{m} / \mathrm{s}$ or $\mathrm{kPa}$.

Many studies have compared testicular damage arising from different operating techniques. In these studies, testicular damage was compared with reference to sexual function, testicular volume and hormone levels $[7,27,28]$. For IH repair surgery, especially with respect to surgical techniques using mesh, there are studies that support [7$9,21]$ or do not $[19,27,29]$ the testicular damage caused by a marked foreign body inflammatory and immunological response to the mesh. In our hospital, mainly Lichtenstein, Kugel and TEP IH repair surgery techniques are used. When these three surgical techniques were compared, no significant difference was found between testicular volume and 2D-SWE values and, therefore, the degree of testicular damage. In addition, there was no significant difference between the testicular volume and 2D-SWE values between operations on direct or indirect hernia types.

There are some limitations in our study. In this study, we included patients who had undergone an IH operation. If we examined the testicles at regular intervals before and after the operation with SWE, we could separately evaluate the possible effects of IH and IH operation on testicular damage; this may be the subject of future re- 
search. A biopsy was not performed in any of the patients due to potential complications. Since the patients did not present with infertility complaints, laboratory tests (e.g., semen analysis, hormone levels) were not requested. All examinations were performed by an experienced radiologist, and this study did not evaluate the possibility of interobserver variability.

In conclusion, our study suggests that the 2D-SWE technique can be effectively used as an imaging method for evaluating testicular damage, using objective numerical values in patients with postoperative IH. Long-term follow-up studies with a larger number of patients are required to confirm the efficacy of using 2D-SWE for routine evaluation in this patient group.

\section{Conflict of interest: none}

\section{References}

1. Ballas $\mathrm{K}$, Kontoulis T, Skouras $\mathrm{CH}$, et al. Unusual findings in inguinal hernia surgery: Report of 6 rare cases. Hippokratia 2009;13:169-171.

2. Beddy P, Ridgway PF, Geoghegan T, et al. Inguinal Hernia Repair Protects Testicular Function: A Prospective Study of Open and Laparoscopic Herniorraphy. J Am Coll Surg 2006;203:17-23.

3. Kumar S, Dikshit P. Effect of bilateraly Lichtenstein hernia repair on male gonadal function: A prospective study. Ind J Sci Res Tech 2015;3:66-71.

4. Ozdamar MY, Karakus OZ. Testicular ischemia caused by incarcerated inguinal hernia in infants: incidence, conservative treatment procedure, and follow-up. Urol J 2017; 14:4030-4033.

5. Bittner R, Schwarz J. Inguinal hernia repair: current surgical techniques. Langenbecks Arch Surg 2012;397:271-282.

6. Bulus H, Dogan M, Tas A, Agladioglu K, Coskun A. The effects of Lichtenstein tension-free mesh hernia repair on testicular arterial perfusion and sexual functions. Wien Klin Wochenschr 2013;125:96-99.

7. Hallen M, Westerdahl J, Nordin P, Gunnarsson U, Sandblom G. Mesh hernia repair and male infertility: A retrospective register study. Surgery 2012;151:94-98.

8. Hallen M, Sandblom G, Nordin P, Gunnarsson U, Kvist U, Westerdahl J. Male infertility after mesh hernia repair: A prospective study. Surgery 2011;149:179-184.

9. Kolbe T, Hollinsky C, Walter I, Joachim A, Rulicke T. Influence of a new self-gripping hernia mesh on male fertility in a rat model. Surg Endosc 2010;24:445-461.

10. Sucullu I, Filiz AI, Sen B, et al. The effects of inguinal hernia repair on testicular function in young adults: a prospective randomized study. Hernia 2010;14:165-169.

11. Durmaz MS, Sivri M, Sekmenli T, Kocaoğlu C, Çiftci İ. Experience of using shear wave elastography imaging in evaluation of undescended testes in children: Feasibil- ity, reproducibility, and clinical potential. Ultrasound Q 2018;34:206-212.

12. Hattapoğlu S, Göya C, Arslan S, et al. Evaluation of postoperative undescended testicles using point shear wave elastography in children. Ultrasonics 2016;72:191-194.

13. Bansal VK, Krishna A, Manek P, et al. A prospective randomized comparison of testicular functions, sexual functions and quality of life following laparoscopic totally extra-peritoneal (TEP) and trans-abdominal pre-peritoneal (TAPP) inguinal hernia repairs. Surg Endosc 2017;31:1478-1486.

14. Lal P, Bansal B, Sharma R, Pradhan G. Laparoscopic TEP repair of inguinal hernia does not alter testicular perfusion. Hernia 2016;20:429-434.

15. Aguilar-García J, Cano-González HA, Martínez-Jiménez MA, de la Rosa-Zapata F, Sánchez-Aguilar M. Unilateral Lichtenstein tension-free mesh hernia repair and testicular perfusion: a prospective control study. Hernia 2018;22:479482.

16. Akbulut G, Serteser M, Yücel A, et al. Can laparoscopic hernia repair alter function and volume of testis? Randomized clinical trial. Surg Laparosc Endosc Percutan Tech 2003;13:377-381.

17. Chan MS, Teoh AY, Chan KW, Tang YC, Ng EK, Leong HT. Randomized double-blinded prospective trial of fibrin sealant spray versus mechanical stapling in laparoscopic total extraperitoneal hernioplasty. Ann Surg 2014;259:432437.

18. El-Awady SE, Elkholy AA. Beneficial effect of inguinal hernioplasty on testicular perfusion and sexual function. Hernia 2009;13:251-258.

19. Ramadan SU, Gokharman D, Tuncbilek I, et al. Does the presence of mesh have an effect on the testicular blood flow after surgical repair of indirect inguinal hernia? J Clin U1trasound 2009;37:78-81.

20. Basha MAA, Saber S, El-Hamid M Abdalla AA, et al. Assessment of the testicular vascularity after inguinal herniotomy in children: a prospective color Doppler study. Acta Radiol 2020;61:128-135.

21. Stula I, Druzijanic N, Srsen D, et al. Influence of inguinal hernia mesh repair on testicular flow and sperm autoimmunity. Hernia 2012;16:417-424.

22. Nath P, Dey S, Karim T, Jain A, Katiyar VK, Patel G. Study of testicular perfusion after Lichtenstein hernioplasty in uncomplicated inguinal hernia. Int Surgy J 2018;5:1104-1110.

23. Erdogan H, Durmaz MS, Arslan S, et al. Shear Wave Elastography Evaluation of Testes in Patients with Varicocele. Ultrasound Q 2020;36:64-68.

24. Zaharko VP, Nakonechnyy AY, Fedus VR. The significance of qualitative compressive elastography in boys with hernia inguinale. Pharma Innovation 2016;5:26-28.

25. Durmaz MS, Arslan S, Özbakır B, et al. Effectiveness of Shear Wave Elastography in the diagnosis of acute pancreatitis on admission. Med Ultrason 2018;20:278284.

26. Youk JH, Son EJ, Park AY, Kim JA. Shear-wave elastography for breast masses: local shear wave speed $(\mathrm{m} / \mathrm{sec})$ versus Young modulus (kPa). Ultrasonography 2014;33:34-39. 
27. Aydede H, Erhan Y, Sakarya A, Kara E, Ilkgul O, Can M. Effect of mesh and its location on testicular flow and spermatogenesis in patients with groin hernia. Acta Chir Belg 2003;103:607-610.

28. Palabiyik FB, Cimilli T, Kayhan A, Toksoy N. Do the manipulations in pediatric inguinal hernia operations affect the vascularization of testes? J Pediatr Surg 2009;44:788790.

29. Çelebi S, Yıldız A, Üçgül A, et al. Do open repair and different laparoscopic techniques in pediatric inguinal hernia repairs affect the vascularization of testes? J Pediatr Surg 2012;47:1706-1710. 